\title{
EXTENDED X-RAY BREMSSTRAHLUNG ISOCHROMAT FINE STRUCTURE OF $\mathrm{SiO}_{2}$
}

\author{
E. SobczaK and R. NiEtubyć \\ Institute of Physics, Polish Academy of Sciences \\ Al. Lotników 32/46, 02-668 Warszawa, Poland \\ (Received August 1, 1994; in final form November 4, 1994) \\ X-ray bremsstrahlung isochromat of amorphous $\mathrm{SiO}_{2}$ deposited on $\mathrm{Si}$ \\ crystal was measured in an energy range up to $250 \mathrm{eV}$ above the threshold. \\ Extended X-ray bremsstrahlung isochromat fine structure (EXBIFS) was \\ observed up to $150 \mathrm{eV}$ for $\mathrm{SiO}_{2}$ studied. The Fourier transform of EXBIFS \\ showed two peaks originated from first and second neighbors around silicon \\ and oxygen ions. Model calculations of EXBIFS of amorphous $\mathrm{SiO}_{2}$ were \\ performed in terms of single scattering of spherical waves and compared \\ with experimental results.
}

PACS numbers: $71.25 . \mathrm{Tn}, 79.20 . \mathrm{Kz}$

\section{Introduction}

One of the most fundamental information needed to understand the various properties of materials is the structural arrangement of the constituent atoms. In general, there is no unique set of atomic coordinates to describe the structure of amorphous solids and the structure is usually characterized by short-range order such as nearest-neighbor distances and coordination numbers, as well as bond angle of coordinated atoms. The radial distribution function (RDF) for amorphous solids is usually measured by using X-ray, neutron and electron diffraction.

Quite another technique of extended X-ray absorption fine structure (EXAFS) has been successfully used for local structural studies of amorphous materials (e.g. [1, 2]) since 15 years. In EXAFS method, the radial distribution function is extracted from the distribution of unoccupied electron states investigated by X-ray absorption spectrum. There are several other EXAFS-like phenomena [3], for example, extended electron loss fine structure (EXELFS), extended appearance potential fine structure (EAPFS) and extended X-ray bremsstrahlung isochromat fine structure (EXBIFS). The latter one has been applied in the present work.

$\mathrm{X}$-ray bremsstrahlung isochromat method (BIS) was introduced in 1915 by Duane and Hunt [4] for the first experimental evaluation of the Planck constant. 
Determination of $h / e$ by using BIS method was improved by several authors and remained the most accurate method for evaluation of Planck's constant until Josephson's experiment in 1969. Since forties the BIS spectroscopy has been used for investigations of electronic structure of solids (for more details on BIS spectroscopy see review papers $[5,6])$.

However, there are still numerous experimental and theoretical problems involved in the BIS spectroscopy, which are not yet solved, e.g. experimental and theoretical investigations of extended X-ray bremsstrahlung isochromat fine structure. Up to now the EXBIFS has been observed for Ge [7] and several metals like $\mathrm{Cu}, \mathrm{Pd}, \mathrm{Ni}, \mathrm{Co}, \mathrm{Ag}, \mathrm{Fe}$ and Mo [8-12] and compared with $K$-edge EXAFS's of these elements. The EXBIFS has been established as a new method of evaluation of the interatomic distances, at least for one component material. It was pointed out that standard procedures developed already for EXAFS analysis can be also used in EXBIFS analysis.

A new question arises, if any EXBIFS structure occurs in a two-component material and how it is related to interatomic distances. The BIS studies of $\mathrm{SiO}_{2}$ in an usual energy range were presented in our previous paper [13], where we have compiled distributions of electron states of both occupied and unoccupied bands of $\mathrm{SiO}_{2}$ on a common energy scale by using photoemission and X-ray bremsstrahlung isochromat spectroscopies. Here we present first EXBIFS investigation of a two-component material, silicon dioxide.

Amorphous $\mathrm{SiO}_{2}$ (referred hereafter as a-SiO 2 ), being a wide-gap insulator, is of considerable technological and theoretical interest. X-ray and neutron diffraction studies of vitreous silica [14] have clearly established that each of the first three peaks in the atomic distribution represents a single type of atom pairing: $\mathrm{Si}-\mathrm{O}$ pairs with $1.6 \AA$ interatomic separation, $\mathrm{O}-\mathrm{O}$ pairs at $2.6 \AA$ and Si-Si pairs at $3.1-3.2 \AA$. This structure is consistent with the random-network theory first proposed by Zachariasen [15]. There are a few reports concerning the EXAFS studies of $\mathrm{SiO}_{2}$ at the $\mathrm{O} K$ edge [1] and $\mathrm{Si} K$ edge [2].

\section{Experimental}

The a-SiO $\mathrm{S}_{2}$ sample was prepared in the Institute of Electronic Materials Technology in Warsaw. The $\mathrm{SiO}_{2}$ film was deposited on a $\mathrm{Si}(111)$ substrate by r.f. sputtering. The film thickness was about $3000 \AA$. A detailed characterization of the sample and experiment is presented in [13].

The intensity of bremsstrahlung radiation was recorded as a function of voltage on the X-ray tube. A Johann monochromator with $\mathrm{Si}(220)$ bent crystal was set at the photon energy $5415 \mathrm{eV}$. The total spectrometer resolution was $1.8 \mathrm{eV}$. The vacuum in the X-ray tube was of order of $10^{-6} \mathrm{Tr}$. Special care has been undertaken to minimize decomposition of silicon oxide during X-ray bremsstrahlung measurements. The BIS spectra of a-SiO $\mathrm{S}_{2}$ layer were measured with $0.5 \mathrm{eV} / 1 \mathrm{~min}$ steps. A sum of 10 isochromats is shown in Fig. 1. The zero energy was located at the Fermi level, which has been determined from the isochromat of Pd metal, and confirmed by isochromat of thin gold layer evaporated onto a-SiO $\mathrm{S}_{2}$ sample. 


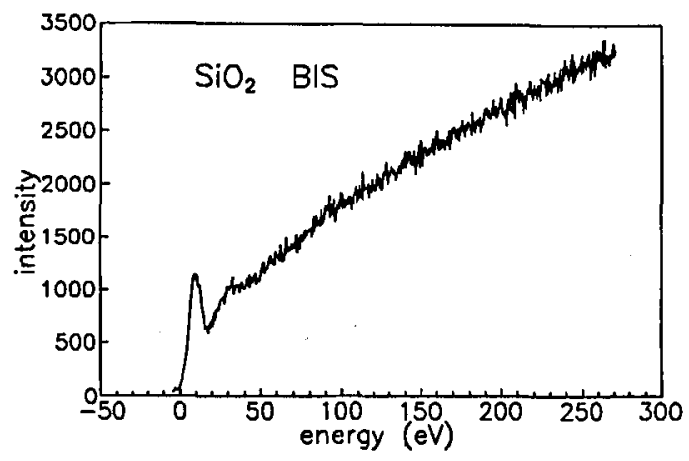

Fig. 1. $\mathrm{SiO}_{2}$ BIS measured in extended range at the $5415 \mathrm{eV}$ photon energy.

\section{Theoretical}

In general, two theoretical approaches are used for theoretical studies of $\mathrm{X}$-ray bremsstrahlung isochromats: electronic band structure and electron scattering calculations. The band structure approach is suitable for the near-threshold BIS, whereas the multiple scattering theory is used for both near and extended energy range $[16,17]$.

It has been established that a single scattering theory in plane wave approximation is very suitable for interpretation of EXBIFS in the case of fcc metals [5]. The normalized EXBIFS function is defined as $\chi(k) \equiv\left(I-I_{0}\right) / I_{0}$, where $I$ is the observed BIS intensity for the material, $I_{0}$ is a smooth BIS contribution from an isolated atom. In this work we tried to calculate EXBIFS of $\mathrm{a}_{-} \mathrm{SiO}_{2}$ in the frame of single scattering theory [5] and spherical waves approximation [18] by using the following formula:

$$
\begin{aligned}
\chi(k) & =\sum_{z, j, l} \frac{N_{z, j}}{k r_{z, j}^{2}}\left|f_{j}\left(k, r_{z, j}, \pi\right)\right| \exp \left(-2 \sigma_{z, j}^{2} k^{2}-2 r_{z, j} / \lambda\right) \\
& \times P_{z, l}^{\text {at }} \sin \left\{2 k r_{z, j}+2 \delta_{z, l}(k)+\theta_{j}\left(k, r_{z, j}\right)+\left[1-(-1)^{l}\right] \pi / 2\right\},
\end{aligned}
$$

where $z$ denotes either Si or $\mathrm{O}$ atom, $\left\{f_{j}\left(k, r_{z, j}, \pi\right)\right\}$ and $\theta_{j}\left(k, r_{z, j}\right)$ are the curve wave backscattering amplitude and phase functions of the $j$-th neighboring atom. The $z$-th partial sum is over shells containing $N_{z, j}$ number of $j$-th atoms at distance $r_{z, j}$ from the $z$-th central atom. $2 \delta_{z, l}(k)+\theta_{j}\left(k, r_{z, j}\right)$ is the $k$-dependent phase shift containing contributions from both the central and scattering atoms, and $\sigma_{z, j}$ is the Debye-Waller factor being a mean-squared relative displacement of the atoms arising from both static and dynamic disorder. The damping parameter $1 / \lambda$ is associated with inelastic scattering of electrons.

The important quantities here are the $l$-partial probabilities $P_{z, l}^{\text {at }}$ of bremsstrahlung transitions in the $z$-th atom. We calculated energy-dependent X-ray bremsstrahlung cross-sections for silicon and oxygen atoms (see Fig. 2) in Coulombic approximation by using tabulated data by Hahn and Rule [19] and a scaling procedure proposed by them. The relative probabilities of X-ray bremsstrahlung transitions to final electron states $\mathrm{O} 3 s, \mathrm{O} 3 p, \mathrm{Si} 3 s, \mathrm{Si} 3 p$ and $\mathrm{Si} 3 d$ for photon 


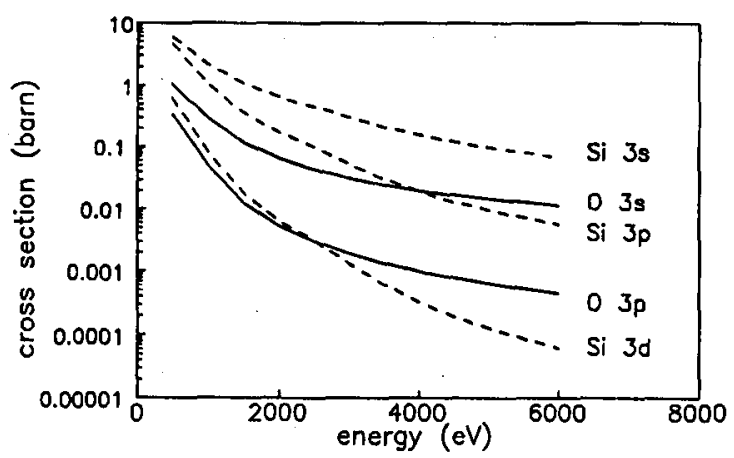

Fig. 2. Contributions of various $n l$-subshells to the cross-section for bremsstrahlung transition in $\mathrm{Si}$ and $\mathrm{O}$ ions derived from paper of Hahn and Rule [19].

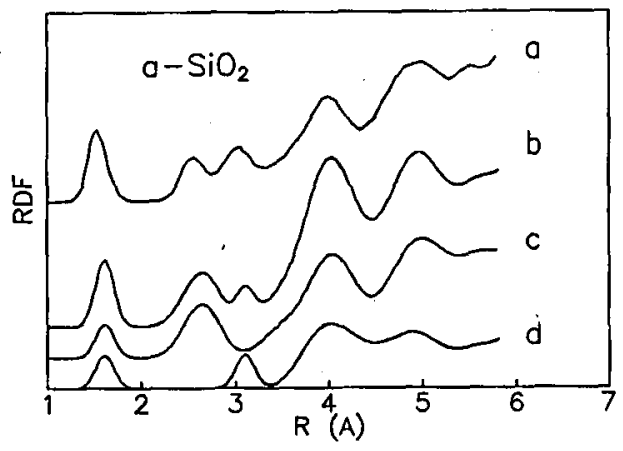

Fig. 3. Radial distribution function for atomic structural models of amorphous $\mathrm{SiO}_{2}$ : (a) X-ray scattering curve from a quasiperiodic model by Ching [21], (b) RDF model of this work. Partial distribution functions around oxygen and silicon ions of the latter model are shown as curves (c) and (d), respectively.

energy $5415 \mathrm{eV}$ are equal to $0.16,0.01,1.0,0.09$ and 0.001 , respectively. This quite simple theory predicts that the probability of BIS transitions to Si $s$-like states dominates over others.

Central atom phase shifts $\delta_{z, l}(k)$ and backscattering functions $f_{j}\left(k, r_{z, j}, \pi\right)$ were calculated for isolated $\mathrm{Si}$ and $\mathrm{O}$ ions in the same way like in paper [20]. A simple model of network for a-SiO ${ }_{2}$ was constructed by taking interatomic distances characteristic of $\beta$-quartz and assuming that the Debye-Waller factor is a progressing function of neighbor shell radius in the range $0.1-0.25 \AA$. The radial distribution function (RDF) obtained for our model network of a-SiO ${ }_{2}$, shown in Fig. 3b, is consistent with RDF of a model network of amorphous $\mathrm{SiO}_{2}$ (Fig. 3a) from the literature [21].

The radial distribution function for the nearest neighbors is dominated by the $\mathrm{Si}-\mathrm{O}$ and $\mathrm{O}-\mathrm{O}$ distances of the $\mathrm{SiO}_{4}$ unit, with the values of $1.62 \AA$ and about $2.6 \AA$, respectively. Atomic distributions around oxygen and silicon ions in a-SiO are given in Fig. 3c and 3d. It is clearly visible that the high maximum of RDF at 


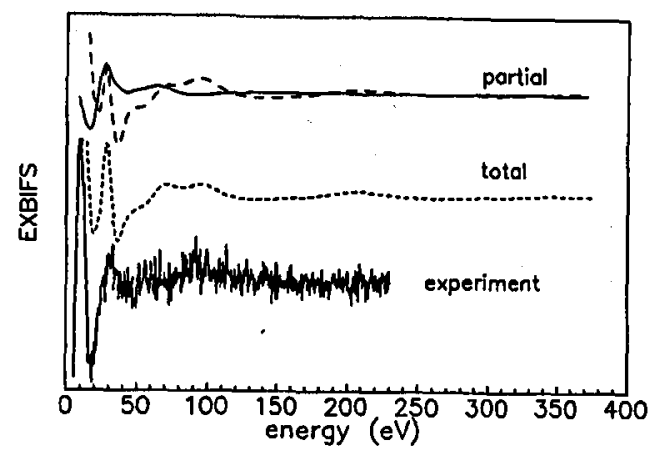

Fig. 4. Experimental EXBIFS of $\mathrm{SiO}_{2}$ is compared with theoretical model EXBIFS of amorphous $\mathrm{SiO}_{2}$ (short dashes). Oxygen (solid line) and silicon (long dashes) contributions to EXBIFS are shown in the upper part of the figure.

the distance of $2.6 \AA$ occurs around oxygen ion, whereas there is an empty space around silicon ion for bond lengths between 1.62 and $3.1 \AA$.

The calculated total EXBIFS of a-SiO ${ }_{2}$ and partial oxygen and silicon contributions are shown in Fig. 4. Theoretical EXBIFS amplitudes are highly damped with energy due to the decrease in backscattering amplitudes with energy, for both $\mathrm{Si}$ and $\mathrm{O}$ ions [20]. It is seen that the total EXBIFS is almost identical with the partial contribution around silicon ions. It should be also pointed out that oscillations of oxygen spectrum are out of phase in comparison with oscillations of silicon spectrum, except for the resonance peak at energy of about $30 \mathrm{eV}$, which originates from both types of atoms.

\section{Results and discussion}

The experimental EXBIFS is shown in the bottom, in Fig. 4. One can see an extended structure of X-ray bremsstrahlung isochromat of a-SiO ${ }_{2}$ up to about $150 \mathrm{eV}$, which consists of four maxima at energy of about 10,30,60 and $90 \mathrm{eV}$ above the threshold. The theoretical EXBIFS agrees poorly with the experimental one. The first maximum of experimental curve corresponds to a "white line" in $\mathrm{X}$-ray absorption spectra at $K$-edges $[1,2]$. We interpret the first maximum as caused mainly by multiple scattering of electrons between the nearest neighbors $\mathrm{Si}-\mathrm{O}$. The multiple scattering is enormously strong in this energy range because of high values of backscattering amplitudes for both oxygen and silicon ions. This state is related to an antibonding state in the molecular theory.

From the theory mentioned above, the EXBIFS spectrum of $\mathrm{SiO}_{2}$ can be well approximated by one contribution, namely $\mathrm{Si} 3 s$. Therefore the Fourier transform of $\chi$ should give as a result the radial structure function, modified by the phase shift and amplitude functions. The EXBIFS features up to about $150 \mathrm{eV}$ above the threshold are plotted in Fig. 5a after a standard $k$-space conversion procedure.

In this work the Fourier transform is calculated in the limited range from $k_{\min }$ to $k_{\max }$ by using the following formula:

$$
g(R)=(2 / \pi)^{1 / 2} \int_{k_{\min }}^{k_{\max }} w(k) k^{n} \chi(k) \mathrm{e}^{\mathrm{i} 2 k R} \mathrm{~d} k,
$$




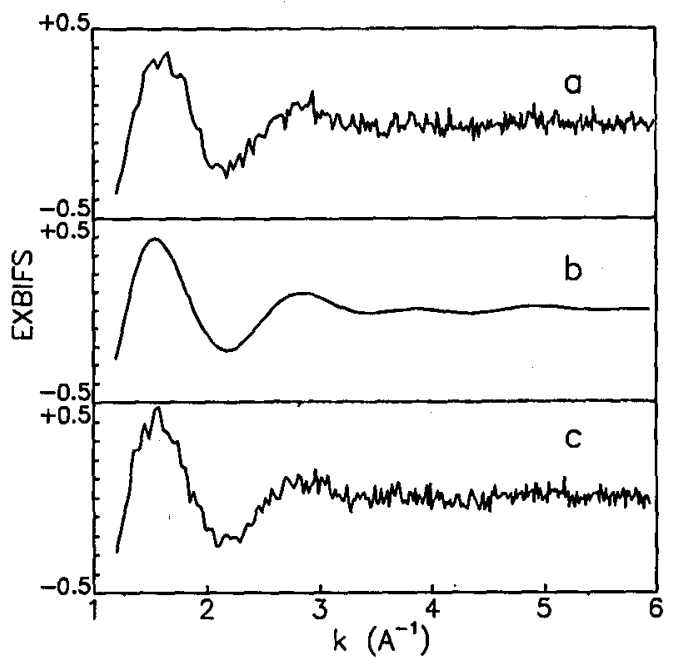

Fig. 5. EXBIFS of amorphous $\mathrm{SiO}_{2}$ shown as function of momentum: (a) experimental curve, (b) an experimental curve after filtering in the $R$ range $0.5-4.0 \AA$, (c) a sum of the smooth curve (b) and a noise generated by computer simulation.

where $w(k)$ is a window function of Gaussian form, and $k^{n}$ is a weighting function with $n=3$. The lower and upper limits of the Fourier integral were chosen at a $k$ value of $1.2 \AA^{-1}$ and $6 \AA^{-1}$, respectively, due to a strong dumping of the $\mathrm{SiO}_{2}$ EXBIFS oscillations as a result of Debye-Waller's effects and low backscattering amplitudes at higher $k$ values.

Since the structure near the edge is highly influenced by multiple scattering and phase shifts are not linear for low $k$ values, a formal single-scattering analysis is not applicable in the near edge range for extracting absolute phase shifts and amplitudes. However, the Fourier transform (FT) performed over the range 1.2-6.0 $\AA^{-1}$ and shown in Fig. $6 \mathrm{a}$ has given us a clear picture of the first and second-shells contributions at about 1.6 and $2.9 \AA$. The first peak corresponds to $\mathrm{Si}-\mathrm{O}$ distance at $1.6 \AA$. The second peak with a value $2.9 \AA$ is in between the $\mathrm{O}-\mathrm{O}$ distance at $2.6 \AA$ and $\mathrm{Si}-\mathrm{Si}$ distance at $3.1 \AA$ shown in RDF in Figs. $3 \mathrm{c}$ and $3 \mathrm{~d}$, therefore we interpret the second FT as caused by second neighbors around both oxygen and silicon ions.

The statistical noise of the BIS measurement plays an important role in limiting the accuracy of the physical quantities obtained from the Fourier analysis of EXBIFS. We evaluated the influence of the noise by using computer simulation. An ideal EXBIFS spectrum (Fig. 5b) was taken as an EXBIFS spectrum, which had been Fourier filtered in the $R$ range from 0.5 to $4.0 \AA$. The ideal spectrum is limited to the first and second shells and its Fourier transform is shown in Fig. $6 \mathrm{~b}$. The noise having a Gaussian distribution of amplitudes according to experimental conditions was generated by computer and added to the ideal spectrum. An example of such a noisy spectrum is shown in Fig. 5c. This procedure was repeated 25 times. 


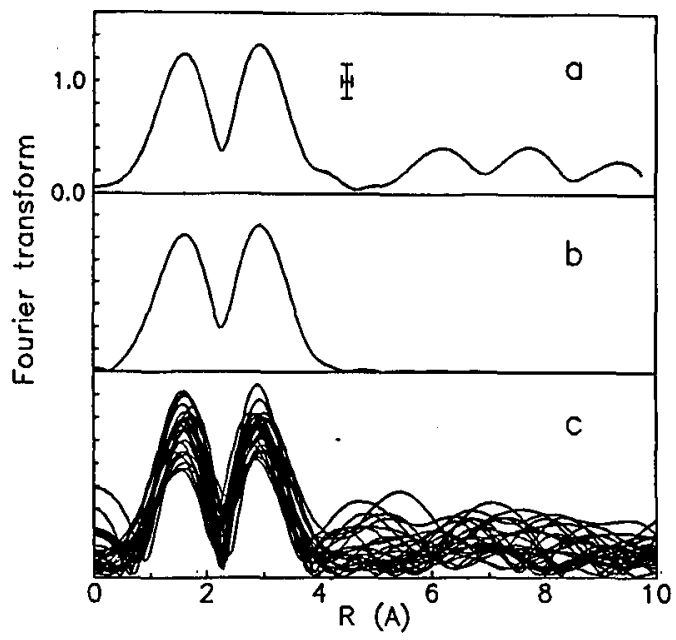

Fig. 6. Moduli of Fourier transforms of $\mathrm{SiO}_{2}$ EXBIFS curves: (a) for experimental spectrum in Fig. 5a, (b) for a smooth curve in Fig. 5b, (c) for 25 spectra obtained in the same way as spectrum in Fig. 5c.

The Fourier transforms of noisy spectra are shown in Fig. 6c. The mean values $\bar{R}$ and $\bar{y}$ of the position and intensity of the Fourier peaks agree well with those of the ideal spectrum. We evaluated the functional standard deviations $\Delta R$ and $\Delta y$ for the Fourier peaks in Fig. 6c. The analysis shows that the experimental noise of our BIS measurement contributes little to an error of interatomic distances by value $\Delta R=0.09 \AA$ and hardly to an error of the Fourier transform magnitude by value $\Delta y=0.15$, which is $13 \%$ of the Fourier peak height. The results clearly indicate that the Fourier transform of experimental EXBIFS for $\mathrm{SiO}_{2}$ at $R$ values bigger than $4 \AA$ is dominated by statistical noise of BIS measurement and cannot be interpreted as caused by next neighbors distances.

\section{Summary}

In this paper we have presented the results of first experimental and theoretical studies of the extended X-ray bremsstrahlung isochromat fine structure for amorphous $\mathrm{SiO}_{2}$ layer, which can provide insight into the nature of bremsstrahlung radiation and relation between the distribution of unoccupied electron states and local atomic structure of $\mathrm{a}_{-} \mathrm{SiO}_{2}$.

Extended X-ray bremsstrahlung isochromat fine structure has been observed up to $150 \mathrm{eV}$ for $\mathrm{SiO}_{2}$ studied. The Fourier transform of EXBIFS over the $k$ range $1.2-6 \AA^{-1}$ has given a clear evidence of the first and second shells contributions at about 1.6 and $2.9 \AA$. The first FT peak corresponds to $\mathrm{Si}-\mathrm{O}$ distance and the second peak corresponds to both the $\mathrm{O}-\mathrm{O}$ and $\mathrm{Si}-\mathrm{Si}$ distances.

The theoretical EXBIFS of $\mathrm{SiO}_{2}$ calculated in free ion approximation with assumption of an amorphous structure of $\mathrm{SiO}_{2}$, based on nearest neighbors distances of $\beta$-quartz, describes qualitatively the experimental EXBIFS of amorphous $\mathrm{SiO}_{2}$ layer deposited on $\mathrm{Si}$. 


\section{Acknowledgment}

This work was supported by the project No. 2P302 25604 of the State Committee for Scientific Research (Republic of Poland).

\section{References}

[1] J. Stöhr, L. Johansson, I. Lindau, P. Pianetta, Phys. Rev. B 20, 664 (1979).

[2] G.N. Greaves, A. Fontaine, P. Lagarde, D. Raoux, S.J. Gurman, Nature 293, 611 (1981).

[3] E.A. Stern, J. Phys. (France) C8, suppl. 12, 47, C8-3 (1986).

[4] W. Duane, F.L. Hunt, Phys. Rev. 6, 166 (1915).

[5] E. Sobczak, Acta Phys. Pol. A 83, 135 (1993).

[6] J.C. Fuggle, in: Unoccupied Electronic States, Eds. J.C. Fuggle, J.E. Inglesfield, Springer-Verlag, Berlin 1992, p. 307.

[7] E. Sobczak, R. Goldberg, J. Pełka, J. Auleytner, in: Inner-Shell and X-Ray Physics of Atoms and Solids, Eds. D.J. Fabian, H. Kleinpoppen, L.M. Watson, Plenum, New York 1981, p. 529.

[8] W. Speier, J.C. Fuggle, R. Zeller, M. Campagna, in: EXAFS and Near Edge Structure III, Eds. K.O. Hodgson, B. Hedman, J.E. Penner-Hahn, Springer, Berlin 1984, p. 496.

[9] W. Speier, T.M. Hayes, J.W. Allen, J.B. Boyce, J.C. Fuggle, M. Campagna, Phys. Rev. Lett. 55, 1693 (1985).

[10] E. Sobczak, J. Auleytner, J. Phys. (France) 48, coll. C9, suppl. 12, 1121 (1987).

[11] E. Sobczak, J. Auleytner, Phys. Rev. B 37, 6251 (1988).

[12] E. Sobczak, J. Auleytner, S. Mobilio, A. Balerna, O. Smotlacha, Physica B 158, 553 (1989).

[13] E. Sobczak, R. Nietubyć, J.W. Sobczak, Acta Phys. Pol. A 86, 837 (1994).

[14] E.H. Henninger, R.C. Buschert, L. Heaton, J. Phys. Chem. Solids 28, 423 (1967).

[15] W. Zachariasen, J. Am. Chem. Soc. 54, 3841 (1932).

[16] A. Simůnek, O. Šipr, J. Vackáŕ, Phys. Rev. Lett. 63, 2076 (1989).

[17] O. Sipr, J. Vackár̆, A. Simůnek, Phys. Rev. B 44, 4832 (1991).

[18] A.G. McKale, G.S. Knapp, S.-K. Chan, Phys. Rev. B 33, 841 (1986).

[19] Y. Hahn, D.W. Rule, J. Phys. B, At. Mol. Phys. 10, 2689 (1977).

[20] R. Nietubyć, Acta Phys. Pol. A 86, 741 (1994).

[21] W.Y. Ching, Phys. Rev. B 26, 6610 (1982). 